\title{
RELACIÓN ENTRE PARÁMETROS DE PRODUCCIÓN Y PROPIE- DADES ESTRUCTURALES DE MADERA LAMINADA ENCOLADA DE Eucalyptus grandis
}

\section{RELATIONSHIP BETWEEN MANUFACTURING PARAMETERS AND STRUCTURAL PROPERTIES OF Eucalyptus grandis GLUED LAMINATED TIMBER}

\author{
Laura Moya $^{1, \$}$, Carolina Pérez Gomar ${ }^{2}$, Abel Vega ${ }^{3}$, Alicia Sánchez $z^{4}$, Ignacio Torino $^{5}$, Vanesa Baño ${ }^{6}$
}

\section{RESUMEN}

La oferta actual de maderas uruguayas ha incentivado la producción de vigas de madera laminada encolada sin adaptar la tecnología original destinada a elementos de carpintería. La inexistencia de normativa sobre requisitos de fabricación permite producir y comercializar componentes que siendo no estructurales, son consumidos como tales por usuarios desconocedores de las características del producto. El objetivo del trabajo fue establecer la relación entre los parámetros de fabricación de madera laminada encolada de Eucalyptus grandis y sus propiedades estructurales, adaptando la tecnología en Uruguay. La metodología involucró modificar los parámetros de producción de una empresa y ejecutar ensayos de caracterización. Los parámetros adoptados incluyeron: adhesivo poliuretánico; diente estructural de longitud $15 \mathrm{~mm}$; presión de ensamble de $10 \mathrm{MPa}$ y de $1,2 \mathrm{MPa}$ en uniones dentadas y vigas respectivamente; y control de tiempos de prensado en láminas y vigas. Los resultados de los ensayos mecánicos y de integridad de encolado indicaron que fue posible fabricar madera laminada estructural. Las propiedades de flexión y densidad de las vigas se correspondieron con las respectivas de la clase resistente GL24h de la norma europea. Estos resultados contribuyen a la redacción de una norma uruguaya de madera laminada encolada estructural de Eucalyptus grandis.

Palabras clave: Densidad, madera laminada, fabricación, resistencia, rigidez; uniones dentadas.

\begin{abstract}
The current supply of locally produced wood in Uruguay led carpentry manufacturers to produce glued laminated timber without technology adjustments. Lack of manufacturing standards allows producers to commercialize non-structural products that are consumed by unaware customers about risk of their use in structural applications. The aim of this study was to establish the relationship between manufacturing parameters of glulam made of Uruguayan Eucalyptus grandis, and its structural properties. Methodology involved definition of manufacturing specifications, supervision of manufacturing process and property characterization. Adopted parameters included: structural polyurethane adhesive, $15 \mathrm{~mm}$-long structural tooth, assembly pressure of $10 \mathrm{MPa}$ and 1,2 $\mathrm{MPa}$ for finger-joint and beam respectively, control of adhesive and applied pressure quantities in both lamellas and beams, and control of pressing and curing times. Results of mechanical

\footnotetext{
${ }^{1}$ Profesora Asociada. Facultad de Arquitectura, Universidad ORT Uruguay. Montevideo, Uruguay.

${ }^{2}$ Asistente de investigación y estudiante de maestría. Facultad de Arquitectura, Universidad ORT Uruguay. Montevideo, Uruguay. ${ }^{3}$ Becario de postdoctorado. Instituto de Estructuras y Transporte. Facultad de Ingeniería, Universidad de la República. Montevideo, Uruguay.

${ }^{4}$ Investigadora. Latitud, Fundación LATU, Laboratorio Tecnológico del Uruguay. Montevideo, Uruguay.

${ }^{5}$ Técnico. Latitud, Fundación LATU, Laboratorio Tecnológico del Uruguay. Montevideo, Uruguay.

${ }^{6}$ Profesora Asociada. Instituto de Estructuras y Transporte. Facultad de Ingeniería, Universidad de la República. Montevideo, Uruguay.

•Autor para correspondencia: moya@ort.edu.uy

Recibido: 03.08.2018 Aceptado: 21.12.2018
} 
tests and gluing integrity on lamellas and beams indicated that it was possible to manufacture structural glulam. Bending and density properties corresponded to those of GL24h of European Standard. Results from this study contribute to drafting a Uruguayan standard for Eucalyptus grandis glulam.

Keywords: Density, finger-joint, glulam, manufacturing, stiffness, strength.

\section{INTRODUCCIÓN}

La madera laminada encolada (MLE) se produce en Uruguay desde la década de los '90. Inicialmente la tecnología fue empleada por empresas nacionales para producir elementos de carpintería de Eucalyptus grandis que eran valorados por su aspecto visual y no por sus propiedades estructurales. A partir del incremento de la disponibilidad de madera nacional como consecuencia de la cosecha de las primeras plantaciones promovidas por la Ley Forestal de 1987, los fabricantes de MLE comenzaron a incluir en su producción vigas y pórticos sin adaptar los procesos de fabricación para la obtención de productos estructurales. En todos los casos, la MLE uruguaya se produce sin ningún marco normativo que regule su fabricación y se comercializa sin cumplir con los requisitos técnicos de un material estructural (Baño et al. 2016).

Tradicionalmente los productos de ingeniería de madera emplean especies de coníferas; tal es así, que la actual norma europea UNE-EN 14080 (AENOR 2013) establece requisitos de fabricación de MLE solamente para madera de coníferas. La redacción de la norma para especies de frondosas se encuentra en el Comité Europeo de Normalización a la espera de información consistente que permita establecer los requisitos de fabricación correspondientes. La generación de datos experimentales relacionados con el comportamiento de la línea de cola y la resistencia de corte para MLE fabricada a partir de la extensa variedad y heterogeneidad de especies de frondosas es una ardua tarea, que ha llevado a países no europeos como Estados Unidos y Japón, a formar grupos de especies y rangos de densidad, para definir los requisitos de fabricación para MLE de frondosas (Aicher et al. 2018). Independientemente de las especies, todas estas normas establecen una serie de parámetros de fabricación dentro de los cuales se incluyen: las características del adhesivo, el diente para la fabricación del endentado de las láminas, la presión aplicada, el contenido de humedad de la madera, y las condiciones climáticas (temperatura y humedad relativa) del recinto de producción.

Los adhesivos para la producción de elementos estructurales están definidos en UNE-EN 14080 (2013), entre los más comúnmente empleados destacan melamina urea formaldehido (MUF), fenol resorcinol (RF), poliuretano monocomponente de curado en húmedo (PUR), y los basados en isocianato y polímeros de emulsión (EPI). Generalmente se aplican para MLE de coníferas ya que su porosidad y capacidad de anclaje del adhesivo implican una mejor adhesión que en las especies frondosas (De Almeida et al. 2014). En Uruguay, la MLE se produce principalmente con Eucalyptus grandis, y adhesivos PUR ó EPI para unir láminas adyacentes.

La geometría del diente, la presión aplicada y su influencia en la aptitud estructural de las uniones dentadas son factores clave en la producción de MLE estructural (Castro y Paganini 1997, Ayarkwa et al. 2000, Bustos et al. 2003, Özcifci y Yapici 2008). Lara-Bocanegra et al. (2017) estudiaron el desempeño mecánico de uniones finger-joint de madera de Eucalyptus globulus producidas con dos geometrías de diente, con longitudes de 10 y $15 \mathrm{~mm}$ de longitud, y encoladas con PUR, y encontraron que el diente de $15 \mathrm{~mm}$ presentó el mejor desempeño en los ensayos de flexión. Las uniones finger-joint indispensables en la unión de bloques para producir vigas de grandes luces, son puntos débiles generados por concentración de tensiones en la zona cercana al diente. Por tanto, sería deseable fabricar láminas con la menor cantidad de uniones dentadas posible; es decir, utilizar bloques de la mayor longitud posible. Contreras et al. (2007) en su trabajo sobre madera laminada de Pinus caribaea reportaron valores superiores de módulo de rotura (34\%) y módulo de elasticidad de flexión (27\%) en vigas construidas con láminas completas, en comparación con los respectivos valores de vigas fabricadas con láminas con uniones finger-joint.

Actualmente ninguna de las fábricas de MLE uruguaya cumple con todos los requisitos mínimos de fabricación de productos estructurales. En particular, las uniones finger-joint se realizan con perfiles y adhesivos (generalmente acetato de polivinilo, PVA) no estructurales y la mayoría de las fábricas no cuenta con control de temperatura y humedad ambiental durante el proceso productivo. Existen antecedentes en Uruguay de vigas de MLE que colapsaron después de 10 años en servicio. La falla se originó en las uniones finger-joint y se propagó a través de las líneas de adhesivo, patología típica asociada al proceso de fabricación (Domenech 
et al. 2016). El objetivo del presente trabajo fue establecer la relación entre los parámetros de fabricación de madera laminada encolada estructural de Eucalyptus grandis y sus propiedades estructurales, adaptando la tecnología disponible en Uruguay. El estudio incluyó la determinación de propiedades mecánicas y la evaluación de la calidad del encolado de la unión dentada derivadas de las modificaciones efectuadas. La información generada en este trabajo será insumo para la redacción de la norma uruguaya de madera laminada encolada.

\section{MATERIALES Y MÉTODOS}

La metodología seguida en el trabajo involucró: i) visitas a fábricas y relevamiento del proceso productivo, ii) ajustes y supervisión del proceso de fabricación, y iii) comprobación experimental del desempeño estructural de la MLE.

\section{Visitas a fábricas y relevamiento del proceso productivo}

$\mathrm{Al}$ inicio del proyecto el equipo investigador realizó visitas a las tres fábricas responsables de la mayor producción nacional de madera laminada encolada, localizadas en los departamentos de Durazno, Rivera y Paysandú. Luego de relevar las condiciones de fabricación y realizar sugerencias preliminares sobre adaptación de los procesos productivos, solamente una de las empresas decidió continuar y participar en el proyecto. Esta empresa siguió instrucciones del equipo investigador, y bajo su supervisión fabricó el material analizado en este estudio.

El proceso de fabricación original de elementos de madera laminada encolada de la empresa consta principalmente de cuatro etapas, diferenciadas por áreas de trabajo, y que se detallan en la Figura 1. Varias de las tareas se realizan en condiciones de fabricación que no cumplen con los requisitos de UNE-EN 14080 (2013). El diagrama presenta superposiciones, e incompatibilidad de tareas que impiden su sectorización en espacios con condiciones controladas de temperatura y humedad relativa del aire.

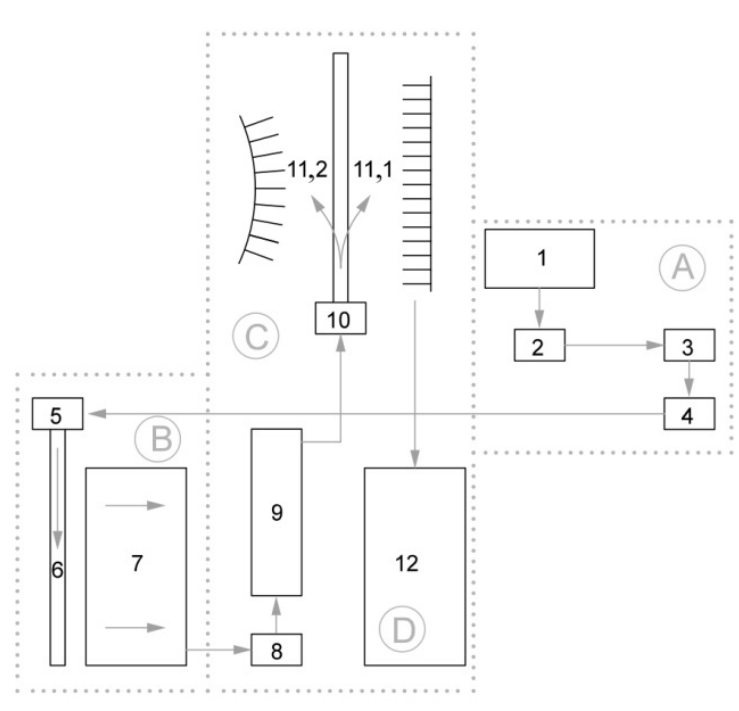

Área A. Preparación de tablas (zona no acondicionada)

\begin{tabular}{cl} 
1. & Acopio de tablas \\
2. & Clasificación y control de $\mathrm{CH}$ \\
3. & Saneado de defectos \\
4. & Apilado de bloques \\
Área B. Fabricación de láminas (zona no acondicionada) \\
\hline
\end{tabular}

$$
\begin{array}{ll}
\text { 5. } & \text { Endentado } \\
\text { 6. } & \text { Presión de endentado } \\
\text { 7. } & \text { Curado del adhesivo en láminas }
\end{array}
$$

Área C. Encolado de vigas (zona no acondicionada)
8. Cepillado de láminas
9. Configuración de vigas
10. Colocación del adhesivo en láminas
11. Prensado de la viga

Área D. Terminación de vigas

12. Cepillado cuatro caras y corte a medida

Figura 1: Layout de la fábrica de la empresa involucrada.

Se constató que: i) la madera empleada en la fabricación de vigas era clasificada visualmente por apariencia, y no por resistencia; ii) la longitud de los bloques componentes de las láminas (limitada por la maquinaria 
empleada) oscilaba entre 200 y $700 \mathrm{~mm}$; iii) la unión entre bloques realizada mediante la técnica finger-joint empleaba un perfil de diente no estructural (12 $\mathrm{mm}$ de longitud $0,35 \mathrm{~mm}$ de ancho, y $3 \mathrm{~mm}$ de paso); iv) el adhesivo PVA era utilizado para las uniones finger-joint; v) la lámina era conformada ubicando los bloques de distintas longitudes en forma aleatoria; vi) la viga era configurada sin verificar que las uniones finger-joint de dos láminas adyacentes no estuviesen alienadas verticalmente en la altura de la viga.

\section{Supervisión y ajustes del proceso de fabricación}

\section{Preparación de bloques y láminas}

De la línea de producción de la fábrica, se seleccionaron al azar 600 tablas de madera aserrada de Eucalyptus grandis de tamaño comercial $(75 \times 26 \times 3300 \mathrm{~mm})$ que habían sido previamente secadas en horno hasta $12 \%$ de contenido de humedad $(\mathrm{CH})$. Las tablas fueron clasificadas visualmente y asignadas a Clase 2 de IRAM 9662-2 (2015), para producir los bloques que conforman las láminas. La longitud máxima de los bloques quedó limitada a $700 \mathrm{~mm}$ por la maquinaria empleada para el aserrado y para el fresado del finger-joint, mientras que la longitud mínima se fijó en $400 \mathrm{~mm}$ (Figura 2). Se eliminaron rajaduras y nudos cercanos al borde de la pieza para evitar en caso de manifestarse en las proximidades de un finger-joint, su debilitamiento estructural. Para ello se estableció una distancia entre el borde del bloque y el nudo más cercano igual o mayor a tres veces el diámetro del nudo (Figura 2).

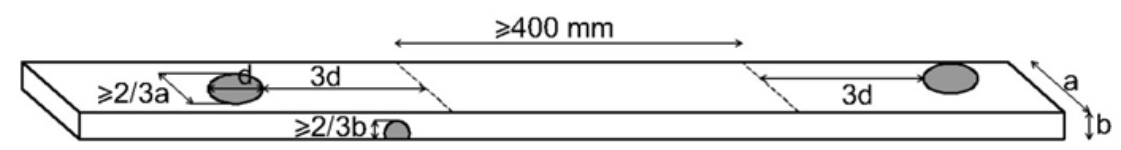

Figura 2: Esquema de saneado de tablas.

La fabricación de la unión finger-joint se realizó de acuerdo a UNE-EN 14080 (2013). Previo a la fabricación de la unión entre bloques, se verificó que éstos presentaran $\mathrm{CH}$ homogéneos, con diferencias iguales o menores a 4\%. La fabricación de la unión se realizó mediante la técnica del endentado y encolado utilizando una fresadora con perfil pluma para endentado de cara, con diente estructural de $15 \mathrm{~mm}$ de longitud y cuya geometría se muestra en la Figura 3.
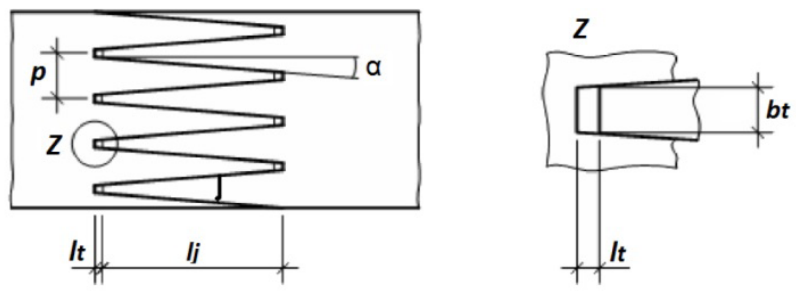

\begin{tabular}{clc}
\hline $\mathbf{l}_{\mathbf{i}}$ & Longitud del diente & $15 \mathrm{~mm}$ \\
\hline $\mathbf{p}$ & Paso & $4 \mathrm{~mm}$ \\
\hline $\mathbf{l}_{\mathbf{t}}$ & Holgura del diente & $0,1 \mathrm{~mm}$ \\
\hline $\mathbf{b}_{\mathbf{t}}$ & Ancho del diente & $0,5 \mathrm{~mm}$ \\
\hline $\mathbf{z}$ & Raíz del diente & \\
\hline
\end{tabular}

Figura 3: Parámetros geométricos del perfil dentado (adaptado de UNE-EN 14080).

El encolado se realizó con adhesivo de poliuretano monocomponente (PUR), aplicado manualmente sobre la superficie de los dientes de las dos testas de los bloques, para conformar las láminas. Sobre las testas de cada lámina se aplicó una presión de ensamblaje de $10 \mathrm{MPa}$ durante $1 \mathrm{~s}$. Luego de ensambladas las láminas fueron retiradas de la prensa, almacenadas durante siete días para el curado del adhesivo, y posteriormente cepilladas 
en sus caras extrayendo aserrín y polvo de la superficie. La temperatura del recinto de fabricación osciló entre $10^{\circ}$ y $13^{\circ} \mathrm{C}$, y la humedad relativa entre 42 y $85 \%$, en las etapas de encolado y fraguado. Cada lámina $(73$ x 24 x $3300 \mathrm{~mm}$ ) fue identificada con un código que indicó pertenencia y posición en relación a la altura de la viga (e.g., V1-01) a efectos de garantizar la configuración planteada de esta última (Figura 4).

\section{Vigas de madera laminada encolada}

Se fabricaron 41 vigas de sección homogénea $(73$ x 192 x $4200 \mathrm{~mm})$ con ocho láminas cada una, y con una configuración adaptada de IRAM 9660-1 (2015). Se evitó dejar un tiempo de poro abierto en las caras de las láminas mayor a $6 \mathrm{~h}$ antes del encolado, y se mantuvo una distancia entre endentados de dos láminas contiguas mayor a $120 \mathrm{~mm}$ (Figura 4). El encolado fue realizado mecánicamente aplicando $300 \mathrm{~g} / \mathrm{cm}^{2}$ (según indicación del fabricante) de modo de distribuir la cola uniformemente sobre la superficie. Las láminas fueron apiladas manualmente en una prensa hidráulica hasta conformar cada una de las vigas.

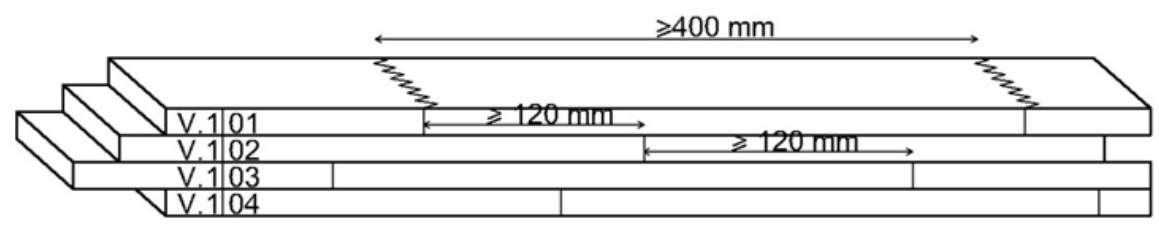

Figura 4: Configuración de vigas.

Posteriormente, se unieron las láminas mediante una prensa hidráulica manteniendo una presión uniforme de 1,2 MPa durante tres horas. Para permitir el curado del adhesivo las vigas fueron almacenadas en un recinto sin condiciones controladas durante siete días, y luego fueron cepilladas hasta sus dimensiones finales (73 x $192 \times 4200 \mathrm{~mm})$.

\section{Comprobación experimental}

La validación del proceso de fabricación y el desempeño estructural de láminas y vigas se realizó a partir de ensayos físicos y mecánicos de acuerdo a normativa europea. Se evaluaron uniones dentadas y vigas laminadas encoladas.

\section{Uniones dentadas (finger-joint)}

La evaluación de los empalmes finger-joint se realizó sobre cuerpos de prueba de $600 \mathrm{~mm}$ de longitud y de sección transversal igual a la de la lámina de la cual fueron extraídos (73 x $24 \mathrm{~mm})$. Se prepararon dos muestras, una con 40 probetas conteniendo un finger-joint en la mitad de su longitud, y otra con 40 probetas de madera sólida (sin finger-joint), que fueron acondicionadas hasta alcanzar 12\% de $\mathrm{CH}$. Los cuerpos de prueba fueron ensayados de cara a flexión en cuatro puntos en máquina universal Minebea con una celda de carga de $50 \mathrm{kN}$ de acuerdo a UNE-EN 408 (2012), y se obtuvo la resistencia de flexión ( $\mathrm{f}_{\mathrm{m}}$ ) con la ecuación provista en UNE-EN 408 (2012).

Luego de finalizado el ensayo, de cada cuerpo de prueba se extrajeron dos rebanadas de $22 \mathrm{~mm}$ de ancho y de igual sección transversal que la probeta, una de cada lado y cercana a la zona de rotura. Se midieron las dimensiones con calibre al $0,01 \mathrm{~mm}$ y se pesaron cada una de ellas al $0,01 \mathrm{~g}$ para calcular la densidad. El $\mathrm{CH}$ se determinó de acuerdo a UNE-EN 13183-1 (2004) por método de secado en estufa (temperatura $100^{\circ} \mathrm{C}$ ).

Los valores característicos de las resistencias de flexión para cuerpos de prueba con unión dentada $\left(f_{m, j, k}\right)$ (Ecuación 1) y para madera sólida $\left(f_{m, l, k}\right)$ (Ecuación 2) fueron determinados considerando una distribución log-normal de acuerdo a UNE-EN 384 (2016) y UNE-EN 14358 (2016):

$$
f_{m, j, k}=\exp \left(\bar{y}-k_{s}(n) s_{y}\right)
$$




$$
f_{m, l, k}=\exp \left(\bar{y}-k_{s}(n) s_{y}\right)
$$

donde $\mathrm{s}_{\mathrm{y}}$ es la desviación estándar; $\mathrm{k}_{\mathrm{s}}(\mathrm{n})$ es el factor de corrección por número de probetas, siendo 1,87 para $\mathrm{n}=40 ;^{\mathrm{y}}$ es el valor medio de la muestra.

\section{Vigas laminadas encoladas}

Las vigas laminadas fueron ensayadas a flexión en cuatro puntos en un pórtico con una celda de carga de $200 \mathrm{kN}$ de acuerdo a UNE-EN 408 (2012). La carga fue aplicada a velocidad constante con un desplazamiento de $0,58 \mathrm{~mm} / \mathrm{s}$ hasta la rotura de la pieza. La resistencia $\left(\mathrm{f}_{\mathrm{m}}\right)$ y el módulo de elasticidad global $\left(\mathrm{E}_{\mathrm{m}, \mathrm{g}}\right)$ se estimaron de acuerdo a UNE-EN 408 (2012). Inmediatamente finalizado el ensayo, de cada viga se extrajo cerca de la zona de rotura, una rebanada libre de defectos de $70 \mathrm{~mm}$ de longitud y de igual sección transversal que la viga (73 x $192 \mathrm{~mm}$ ), para determinar el $\mathrm{CH}$ y la densidad siguiendo los mismos procedimientos descritos para las uniones dentadas.

Los valores del módulo de elasticidad medio $\left(\mathrm{E}_{\mathrm{m}, \mathrm{g}}\right)$ y la densidad fueron ajustados a las condiciones de referencia según UNE-EN 384 (2016). El valor característico de la resistencia de flexión $\left(\mathrm{f}_{\mathrm{m}, \mathrm{k}}\right.$ ) determinado mediante la Ecuación 3 y conforme con UNE-EN 384 (2016), fue corregido por altura de la viga $\left(\mathrm{k}_{\mathrm{h}}\right)$ y por espesor de lámina (k) mediante la Ecuación 4 de acuerdo a UNE-EN 14080 (2013), para obtener la resistencia global de flexión $\left(\mathrm{f}_{\mathrm{m}, \mathrm{g}, \mathrm{k}}\right)$ :

$$
f_{m, k}=\exp \left(\bar{y}-k_{s}(n) s_{y}\right)
$$

donde $\mathrm{s}_{\mathrm{y}}$ es la desviación estándar; $\mathrm{k}_{\mathrm{s}}(\mathrm{n})$ es el factor de corrección por número de probetas, siendo 1,87 para $\mathrm{n}=41$; es el valor medio de la muestra.

$$
f_{m, g, k}=f_{m, k} k_{n} k
$$

El desempeño de la línea de adhesivo se realizó mediante ensayos de delaminación de acuerdo a UNE-EN 14080 (2013). De cada viga se extrajo una probeta libre de defectos, de $70 \mathrm{~mm}$ de longitud y de igual sección transversal que la viga $(73 \times 192 \mathrm{~mm})$. Luego de ser pesadas, las probetas se sumergieron en agua a temperaturas de $10^{\circ} \mathrm{C}-20^{\circ} \mathrm{C}$ en un autoclave, y fueron sometidas al método $\mathrm{C}$ indicado en la norma. Finalizado el ensayo, en cada probeta se midió con regla milimetrada el largo total de la delaminación sobre los planos de encolado. La delaminación total de la probeta y la delaminación máxima para cada línea de cola se determinaron de acuerdo a la Ecuacion 5 y Ecuacion 6, respectivamente:

$$
\begin{gathered}
\operatorname{Delam}_{\text {total }}=l_{\text {tot,delam }} / l_{\text {tot,plano encol }}(\%) \\
\text { Delam }_{\text {max }}=l_{\text {max, delam }} / 2 l_{\text {plano encol }}(\%)
\end{gathered}
$$

donde $l_{\text {tot,delam }}$ es la longitud total de delaminación (en $\mathrm{mm}$ ), $l_{\text {to }}$ encolado en las superficies de las dos testas de cada probeta (en $\mathrm{mm}$ ), $l$ inación (en mm), $l_{\text {plano encol }}$ es la longitud de una línea de cola (en mm). es la longitud total de planos de es la longitud máxima de delam- 


\section{RESULTADOS Y DISCUSIÓN}

\section{Parámetros de producción}

Los parámetros de producción fueron ajustados adaptando la tecnología de la fábrica a los requisitos de UNE-EN 14080 (2013) y se detallan a continuación:

- clasificación visual y definición de longitud mínima de bloques (400 mm);

- empleo de adhesivo PUR estructural en uniones dentadas y láminas;

- adopción de diente estructural de $15 \mathrm{~mm}$ de longitud;

- presión de ensamble de $10 \mathrm{MPa}$ y de 1,2 $\mathrm{MPa}$ en láminas y vigas respectivamente;

- configuración de vigas respetando distancia mínima de uniones $(120 \mathrm{~mm})$ entre láminas adyacentes;

- control de cantidad de adhesivo y presión aplicada en endentados y láminas;

- control de tiempos de poro abierto en uniones dentadas y láminas;

- control de tiempos de prensado y curado.

Los parámetros de temperatura $\left(10^{\circ}-13^{\circ} \mathrm{C}\right)$ y humedad relativa $(42 \%-85 \%)$ del recinto de producción no cumplieron con lo establecido en UNE-EN 14080 para las etapas de encolado ( $\geq 15^{\circ} \mathrm{C}$ y $40-75 \%$ ), y para las etapas de fraguado y acondicionado $\left(\geq 20^{\circ} \mathrm{C}\right.$ y $\left.30 \%\right)$, debido a los costos operativos que conllevaban para la empresa.

\section{Comportamiento de la unión dentada}

Los valores medios y característicos de la resistencia de flexión de las uniones dentadas resultaron inferiores a los correspondientes de la madera (Tabla 1), y son congruentes con el concepto de que la unión dentada constituye un punto crítico de la lámina endentada-encolada. La configuración geométrica de las uniones dentadas posibilita la concentración de tensiones en la zona del endentado debilitando la pieza, e impidiendo alcanzar valores de resistencia similares a los de la madera sólida a pesar de existir correcta adherencia entre las partes ensambladas.

Tabla 1: Resistencia de flexión de uniones dentadas y madera sólida de láminas.

\begin{tabular}{|c|c|c|c|c|c|c|}
\hline \multicolumn{7}{|c|}{ Cuerpos de prueba con unión dentada } \\
\hline $\mathrm{n}$ & $\begin{array}{c}\mathrm{CH} \\
(\%)\end{array}$ & $\begin{array}{c}\text { Densidad } \\
\left(\mathrm{kg} / \mathrm{m}^{3}\right)\end{array}$ & $\begin{array}{c}\mathrm{f}_{\mathrm{m}} \\
(\mathrm{MPa})\end{array}$ & $\begin{array}{c}\text { COV } \\
(\%)\end{array}$ & $\begin{array}{c}\mathrm{f}_{\mathrm{m}, \mathrm{j}, 05} \\
(\mathrm{MPa})\end{array}$ & $\begin{array}{c}\mathrm{f}_{\mathrm{m}, \mathrm{j}, \mathrm{k}} \\
(\mathrm{MPa})\end{array}$ \\
\hline \multicolumn{7}{|c|}{ Cuerpos de prueba sin unión dentada } \\
\hline 40 & 9,64 & 560 & 43 & 42 \\
\hline $\mathrm{n}$ & $\begin{array}{c}\mathrm{CH} \\
(\%)\end{array}$ & $\begin{array}{c}\text { Densidad } \\
\left(\mathrm{kg} / \mathrm{m}^{3}\right)\end{array}$ & $\begin{array}{c}\mathrm{f}_{\mathrm{m}} \\
(\mathrm{MPa})\end{array}$ & $\begin{array}{c}\mathrm{COV} \\
(\%)\end{array}$ & $\begin{array}{c}\mathrm{f}_{\mathrm{m}, 1,05} \\
(\mathrm{MPa})\end{array}$ & $\begin{array}{c}\mathrm{f}_{\mathrm{m}, \mathrm{l}, \mathrm{k}} \\
(\mathrm{MPa})\end{array}$ \\
\hline 40 & 9,70 & 545 & 77 & 19 & 56 & 52 \\
\hline
\end{tabular}

$\mathrm{f}_{\mathrm{m}}$ : Resistencia de flexión, valor medio

$\mathrm{f}_{\mathrm{m}, \mathrm{j}, 05}:$ Resistencia de flexión de uniones dentadas, $5^{\circ}$ percentil

$\mathrm{f}_{\mathrm{m}, \mathrm{j}, \mathrm{k}}:$ Resistencia característica de flexión de las uniones dentadas

$\mathrm{f}_{\mathrm{m}, 1,05}$ : Resistencia de flexión de láminas, $5^{\circ}$ percentil

$\mathrm{f}_{\mathrm{m}, \mathrm{l}, \mathrm{k}}$ : Resistencia característica de flexión de láminas. 
Los tres modos de fallo observados en los ensayos de flexión sobre los cuerpos de prueba con unión dentada fueron (Figura 5): a) por madera, b) mixto, c) por adhesivo.

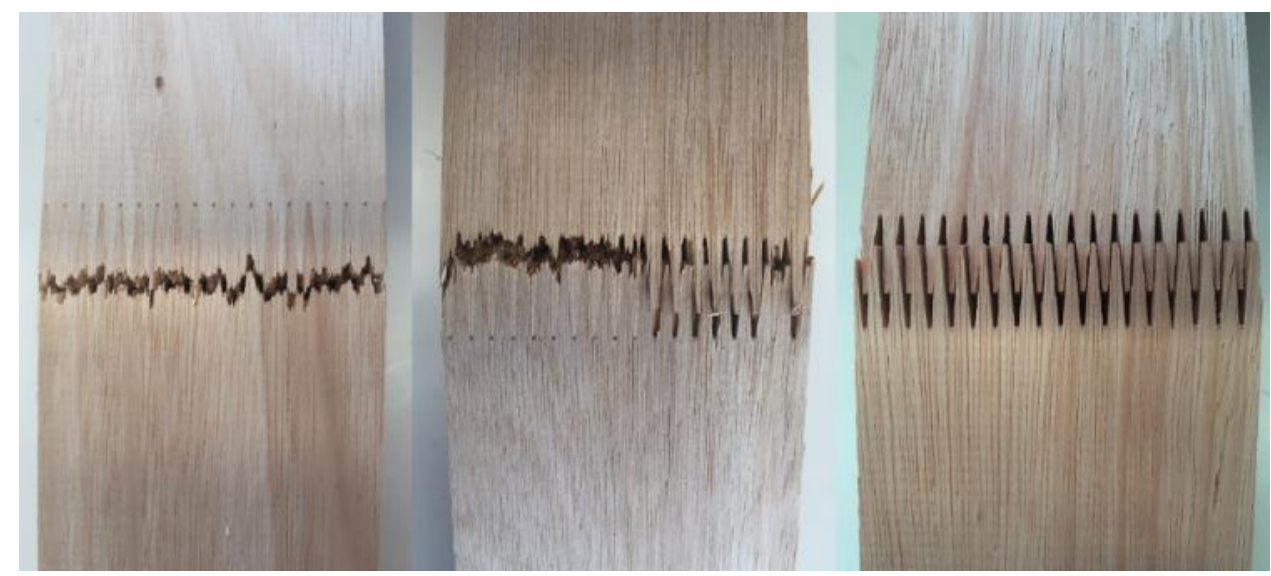

Figura 5: Modos de fallo: a) madera, b) mixto, c) adhesivo.

El modo de fallo a) indica que la resistencia del adhesivo fue mayor que la de la madera, y por tanto, la falla ocurrió en la madera en el tercio central de la probeta, pero no necesariamente en la unión dentada. El modo de fallo b) indica que la rotura se produjo en distintas proporciones tanto en la madera como en la unión dentada por desencolado. El modo de fallo c) muestra que la unión se desencoló por comportamiento deficiente del adhesivo.

Los resultados de resistencia de flexión de uniones dentadas discriminados por modo de fallo, indican que del total de cuerpos de prueba, $75 \%$ fallaron por madera, y $25 \%$ por adhesivo y mixto (Tabla 2). Los valores de resistencia a flexión en el $25 \%$ de las uniones (fallo b y c) fueron superiores a los correspondientes por fallo por madera. Vale señalar que en las probetas con fallo mixto (b), no se discriminaron y no se cuantificaron los porcentajes de fallo por adhesivo y por madera, habiéndose adoptado el criterio más exigente, esto es, adjudicarles el modo de fallo por adhesivo (c). Este criterio parte del supuesto de que el desempeño de la unión fue eficiente cuando el fallo ocurrió por madera, e insuficiente si se produjo por desencolado del endentado.

Tabla 2: Resistencia de flexión de uniones dentadas según el modo de fallo.

\begin{tabular}{cccccccc}
\hline $\mathbf{n}$ & Modo de fallo & $\begin{array}{c}\mathbf{C H} \\
\mathbf{( \% )}\end{array}$ & $\begin{array}{c}\text { Densidad } \\
\left(\mathbf{k g} / \mathbf{m}^{3}\right)\end{array}$ & $\begin{array}{c}\mathbf{f}_{\mathbf{m}} \\
(\mathbf{M P a})\end{array}$ & $\begin{array}{c}\mathbf{C O V} \\
(\mathbf{\%})\end{array}$ & $\begin{array}{c}\mathbf{f}_{\mathbf{m}, \mathbf{j}, 05} \\
(\mathbf{M P a})\end{array}$ & $\begin{array}{c}\mathbf{f}_{\mathbf{m}, \mathrm{j}, \mathrm{k}} \\
(\mathbf{M P a})\end{array}$ \\
30 & a) & 9,64 & 554 & 58 & 18 & 41 & 40 \\
\hline 10 & b) y c) & 9,67 & 581 & 75 & 8 & 67 & 49 \\
\hline
\end{tabular}

a), b), c) Modo de fallo por madera, mixto, por adhesivo, de acuerdo a Figura 4

$\mathrm{CH}$ : Contenido de humedad, valor medio

$\mathrm{f}_{\mathrm{m}}$ : Resistencia a la flexión, valor medio

$\mathrm{f}_{\mathrm{m}, \mathrm{j}, 05}$ : Resistencia a la flexión de las uniones dentadas, $5^{\circ}$ percentil

$\mathrm{f}_{\mathrm{m}, \mathrm{j}, \mathrm{k}}$ : Resistencia característica a flexión de las uniones dentadas.

Resultados similares fueron a reportados por Vega et al. (2017) para láminas de madera de Eucalyptus grandis fabricadas con adhesivos de isocianato y poliuretano, y longitudes de diente de 12 y $15 \mathrm{~mm}$. Si se comparan los valores medios, en vez de los valores característicos que incluyen una penalización por tamaño muestral cuando el número de probetas es inferior a 40 (UNE-EN 384 2010), como es el caso de las 10 probetas que fallaron por adhesivo, se observa que el valor medio de la resistencia de flexión ( $75 \mathrm{MPa})$ es ligeramente inferior al valor medio de las probetas de madera sólida $(77 \mathrm{MPa})$, y significativamente mayor $(\mathrm{p}<0,05)$ 
que el valor medio de la resistencia de las probetas con finger-joint que fallaron por madera (58 MPa). Este resultado puede ser atribuido a la calidad de la madera que conforma las láminas; las probetas de madera con menor resistencia rompen por madera en la zona debilitada por el endentado y antes de agotar la resistencia del sistema adhesivo-madera. Si la madera tiene mayor resistencia, las raíces de los dientes soportan mayores esfuerzos y por tanto se alcanza la resistencia máxima del adhesivo, y se produce el fallo por desencolado del finger. Cabe señalar que este comportamiento se produce cuando no se logra una adhesión eficiente, y los valores de resistencia de la unión encolada son muy cercanos a los valores de resistencia de la madera.

\section{Desempeño mecánico de las vigas de madera laminada encolada}

Los valores de las propiedades de flexión y la densidad de las vigas se muestran en la Tabla 3. Las dimensiones de la sección transversal de las 41 vigas presentaron valores medios de 74,15 mm de base y 193,83 mm de altura, y el $\mathrm{CH}$ exhibió un valor medio de $14,10 \%$ con un COV de $5 \%$.

Tabla 3: Propiedades de flexión y densidad de vigas de madera laminada encolada.

\begin{tabular}{|l|l|}
\hline Resistencia de flexión, valor medio: $\mathrm{f}_{\mathrm{m}, \mathrm{g}, \mathrm{m}}$ & $45 \mathrm{MPa}(\mathrm{COV}=25 \%)$ \\
\hline Resistencia característica de flexión ${ }^{(1)}: \mathrm{f}_{\mathrm{m}, \mathrm{g}, \mathrm{k}}$ & $25 \mathrm{MPa}$ \\
\hline Módulo de elasticidad global, valor medio $^{(2)}: \mathrm{E}_{0, \mathrm{~g}, \mathrm{~m}}$ & $13760 \mathrm{MPa}(\mathrm{COV}=12 \%)$ \\
\hline Densidad característica $^{(2)}: \rho_{\mathrm{g}, \mathrm{k}}$ & $504 \mathrm{~kg} / \mathrm{m}^{3}$ \\
\hline
\end{tabular}

(1)Valor corregido a la altura de referencia de $600 \mathrm{~mm}$ de la viga y al espesor de referencia de $40 \mathrm{~mm}$ de la lámina de acuerdo a UNE-EN 14080 (2013);

${ }^{(2)}$ Valores corregidos al contenido de humedad de referencia de 12\% de acuerdo a UNE-EN 14080 (2013).

Los resultados están en concordancia con los reportados por Piter et al. (2007) para vigas laminadas de Eucalyptus grandis argentino y adhesivo MUF; en particular, con los valores característicos de la resistencia de flexión $\left(\mathrm{f}_{\mathrm{m}, \mathrm{g}, \mathrm{k}}=23,3 \mathrm{MPa}\right)$ y de la densidad $\left(\rho_{\mathrm{g}, \mathrm{k}}=540 \mathrm{~kg} / \mathrm{m}^{3}\right)$ de vigas GL2 compuestas por láminas de madera de la clase de resistencia 2 (inferior), clasificadas de acuerdo a IRAM 9662-2 (2015). Adicionalmente, si se comparan los resultados con los valores de madera laminada encolada de Eucalyptus grandis tabulados en IRAM 9660-1 (2015), se observa que las propiedades de flexión de las vigas laminadas analizadas son similares a las del Grado $2\left(\mathrm{f}_{\mathrm{m}, \mathrm{g}, \mathrm{k}}=21 \mathrm{MPa}\right.$ y $\left.\mathrm{E}_{0, \mathrm{~g}, \mathrm{~m}}=13000 \mathrm{MPa}\right)$ de la mencionada norma.

Los modos de fallo de las vigas laminadas en los ensayos de flexión: fueron: a) por madera, y b) por desencolado de la unión dentada (Figura 6).

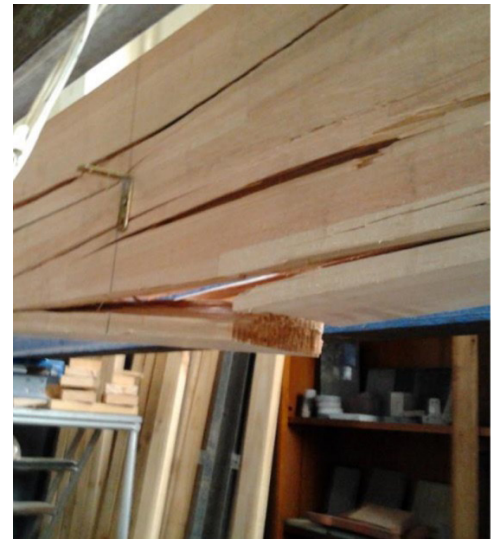

(a)

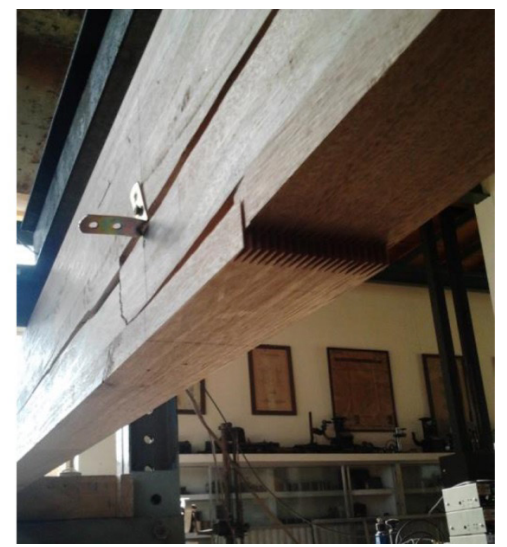

(b)

Figura 6: Modos de fallo de vigas laminadas: a) madera b) adhesivo. 
Los resultados de los ensayos de flexión discriminados por modo de fallo (Tabla 4) indican que el 22\% de las fallas comenzó por desencolado de la unión dentada de las láminas ubicadas en la zona traccionada de la viga (modo de fallo b).

Tabla 4: Resultados de ensayos de flexión de vigas laminadas según el modo de fallo.

\begin{tabular}{cccccc}
\hline $\mathbf{n}$ & $\begin{array}{c}\text { Modo de } \\
\text { fallo }\end{array}$ & $\begin{array}{c}\rho_{\mathbf{g}, \mathbf{k}}{ }^{(2)} \\
\left(\mathbf{k g} / \mathbf{m}^{3}\right)\end{array}$ & $\begin{array}{c}\mathbf{E}_{\mathbf{0}, \mathbf{g , m}}{ }^{(\mathbf{M})} \\
\mathbf{M P a})\end{array}$ & $\begin{array}{c}\mathbf{C O V} \\
\mathbf{( \% )}\end{array}$ & $\begin{array}{c}\mathbf{f}_{\mathbf{m}, \mathbf{g}, \mathbf{k}}(\mathbf{1}) \\
\mathbf{( M p a )}\end{array}$ \\
\hline 9 & $\mathrm{a})$ & 505 & 13708 & 13 & 27,40 \\
\hline & $\mathrm{b})$ & 532 & 14145 & 7 & 31,05 \\
\hline
\end{tabular}

(1)Valor corregido a la altura de referencia de $600 \mathrm{~mm}$ de la viga y al espesor de referencia de $40 \mathrm{~mm}$ de la lámina de acuerdo a UNE-EN 14080 (2013);

${ }^{(2)}$ Valores corregidos al contenido de humedad de referencia de 12\% de acuerdo a UNE-EN 14080 (2013); a), b): fallo por madera y adhesivo respectivamente, de acuerdo a Figura 6

$\rho_{\mathrm{g}, \mathrm{k}}:$ Densidad característica

$\mathrm{E}_{0, \mathrm{~g}, \mathrm{~m}:}$ Módulo de elasticidad global en flexión, valor medio

$\mathrm{f}_{\mathrm{m}, \mathrm{g}, \mathrm{k}}$ : Resistencia característica de flexión.

Las vigas con fallo tipo $\mathrm{b}$ exhibieron valores de resistencia y rigidez mayores que los correspondientes a las vigas con fallos desarrollados en la madera, y son concordantes con los resultados de los ensayos de flexión de las láminas, donde los valores de resistencia de las uniones dentadas (fallo b y c) fueron superiores que los de la madera sólida (Tabla 2). Los porcentajes por modo fallo para las vigas, $78 \%$ en madera, y $22 \%$ por adhesivo, son consistentes con los respectivos porcentajes de fallo de las uniones dentadas en el ensayo de flexión ( $75 \%$ en madera, y $25 \%$ por adhesivo), y muestran la influencia del desempeño de la unión dentada en el de la viga laminada encolada. Vale destacar que las operaciones de control de la producción de MLE incluyen ensayos de los empalmes por unión dentada de láminas, que suelen considerarse como un indicador que anticipa el comportamiento de flexión de las vigas. La razón entre la resistencia de flexión de vigas $\left(\mathrm{f}_{\mathrm{m}, \mathrm{g}, \mathrm{m}}\right)$ y la de las láminas $\left(\mathrm{f}_{\mathrm{m}}\right)$ fue 0,72 .

Adicionalmente, del análisis visual del patrón de fallo en toda su extensión, surge que independientemente del origen, i.e., por desencolado del finger o por rotura de la madera, la falla en la mayoría de las vigas, continuó por fractura de madera en dirección paralela a la fibra, y no por desencolado de láminas adyacentes, sugiriendo un comportamiento de encolado eficiente para la mayoría de las vigas ensayadas.

Finalmente, los valores de las propiedades de flexión y de la densidad al ser comparados con los establecidos para la madera laminada encolada de sección transversal homogénea del sistema europeo de clases resistentes (UNE-EN 14080 2013), permiten la asignación de las vigas a la clase resistente GL24h ( $f_{\mathrm{m}, \mathrm{g}, \mathrm{k}}=24$ $\left.\mathrm{MPa}, \mathrm{E}_{0, \mathrm{~g}, \mathrm{~m}}=11500 \mathrm{MPa}, \rho_{\mathrm{g}, \mathrm{k}}=385 \mathrm{~kg} / \mathrm{m}^{3}\right)$.

\section{Desempeño de la línea de adhesivo en las vigas laminadas}

Luego del ensayo de delaminación, se observaron grietas y deformaciones en las láminas producto de las tensiones de secado (Figura 7). Los resultados del ensayo indicaron que ninguna de las probetas cumplió con los requisitos de UNE-EN 14080 (2013). El porcentaje de delaminación total promedio de los 41 ensayos fue de $42 \%$, superando los máximos admitidos de $4 \%$ para un ciclo de vacío/presión, y de $8 \%$ para el caso de someter las probetas a un segundo ciclo de ensayo. Con respecto al porcentaje máximo de delaminación por línea de cola, 41\% de las líneas de adhesivo fue inferior al máximo de 30\% establecido en UNE-EN 14080 (2013). 

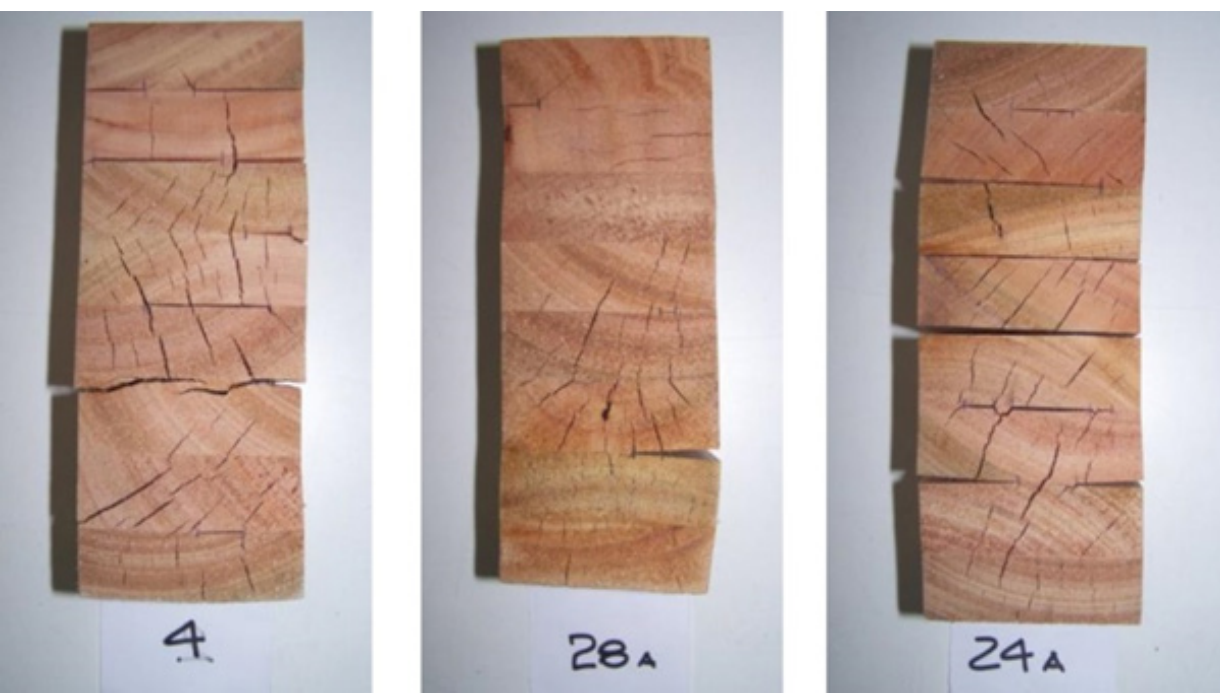

Figura 7: Probetas de madera laminada encolada después del ensayo de delaminación.

Sin embargo, solamente una probeta cumplió con este requisito para la totalidad de sus planos de encolado, i.e., para los siete planos, exhibiendo porcentajes que variaron entre 0 y $27 \%$; el resto de las probetas, superó el porcentaje en alguno de sus planos de encolado. El deficiente comportamiento de adhesión entre láminas puede atribuirse a que no se contempló la indicación de ubicar las láminas con la médula orientada del mismo lado en la sección transversal de la viga. En algunas probetas se observó que las deformaciones en dos láminas contiguas con médulas orientadas en lados opuestos, generaron su desencolado (Figura 7). Otro factor que pudo haber influido en detrimento del desempeño de la unión entre láminas, fue la temperatura ambiente del recinto de encolado y fraguado que osciló entre $10^{\circ}$ y $13^{\circ} \mathrm{C}$, inferior a lo especificado en UNE-EN 14080. Estas temperaturas pudieron haber afectado la viscosidad del adhesivo PUR, que tiende a aumentar con temperaturas bajas, y haber disminuido la cantidad de adhesivo por unidad de superficie aplicada. Resultados similares, superiores al 4\% de delaminación total, fueron reportados por Bourscheid et al. (2015), quienes observaron $34 \%$ de delaminación media total y rajaduras en casos de exposición prolongada a la intemperie, en vigas de madera laminada de Eucalyptus spp. con PUR. Franke y Marto (2014) estudiaron vigas de madera laminada de Eucalyptus globulus con PUR, variando tres parámetros de producción y sus respectivas combinaciones, i.e., dos tiempos de prensado de vigas, cepillado ó lijado de láminas, y empleo ó no de un imprimador; y encontraron que ninguna de las combinaciones generó vigas que cumplieron con los requisitos de UNE-EN 14080 inherentes a integridad y durabilidad de las líneas de adhesivo, pero sí con los de resistencia de flexión. Concluyeron que para condiciones estables de variaciones de contenido de humedad, la mayoría de las combinaciones estudiadas resultaron adecuadas para la producción de vigas laminadas. De Almeida et al. (2014) y Calil et al. (2014) indicaron que en vigas de madera laminada de Lyptus (híbrido de E. grandis y E. urophylla) con adhesivos PUR, RF, ó MUF, los porcentajes de delaminación fueron mayores a los establecidos en la norma NBR 7190 (ABNT 1997), sugiriendo que la dificultad radicó en las propiedades físicas y químicas de la especie y no en el tipo de adhesivo.

\section{CONCLUSIONES}

La propuesta de requisitos de fabricación presentada en este trabajo adaptando la tecnología disponible en Uruguay a las especificaciones del cuerpo normativo europeo, permitieron producir madera laminada encolada de Eucalyptus grandis con características estructurales. 
Los resultados de los ensayos de flexión de las uniones dentadas mostraron valores medios mayores en aquellas uniones que fallaron por adhesivo, que en las que fallaron por madera, indicando un correcto desempeño estructural de las láminas. El endentado-encolado originó una reducción de $24 \%$ en la resistencia de flexión de la lámina, con respecto a la madera sólida sin unión. Por tanto, el procedimiento y los parámetros involucrados en la fabricación de las láminas, tales como compatibilidad madera-adhesivo, mecanizado del endentado, cantidad y modo de aplicación del adhesivo, y presión de prensado, pueden ser considerados aceptables.

Las vigas de madera laminada encolada fabricadas a partir de tablas de la clase resistente 2 de IRAM 9662-2 (2015), adhesivo poliuretánico, y de acuerdo a las condiciones detalladas en este trabajo mostraron resultados experimentales comparables a los valores del Grado 2 para madera laminada de Eucalyptus grandis tabulados en IRAM 9660-1 (2015). Las propiedades de flexión y densidad de las vigas analizadas permitieron su asignación a la clase resistente GL24h de UNE-EN 14080. Los ensayos de delaminación de las vigas indicaron un comportamiento deficiente del sistema adhesivo-adherente en el plano de encolado entre dos láminas adyacentes; y sugieren la necesidad de futuras investigaciones relacionadas con las características de adhesividad de esta especie.

Los resultados de este trabajo contribuyen a la formulación de unos requisitos básicos de producción que servirán de base para la redacción de una norma uruguaya de fabricación de madera laminada encolada.

\section{AGRADECIMIENTOS}

Los autores agradecen a la Agencia Nacional de Investigación e Innovación (ANII), Uruguay, Fondo Innovagro- PR-FSA-2013-1-12897 por los fondos para este proyecto.

\section{REFERENCIAS}

Asociación Española de Normalización y Certificación. UNE. 2016. Madera estructural. Determinación de los valores característicos de las propiedades mecánicas y la densidad. UNE-EN 384. 2010. Asociación Española de Normalización y Certificación: Madrid, España.

Asociación Española de Normalización y Certificación. UNE. 2016. Madera estructural. Determinación de los valores característicos de las propiedades mecánicas y la densidad. UNE-EN 384. 2016. Asociación Española de Normalización y Certificación: Madrid, España.

Asociación Española de Normalización y Certificación. UNE. 2016. Estructuras de madera. Determinación y verificación de los valores característicos. UNE-EN 14358. 2016. Asociación Española de Normalización y Certificación: Madrid, España.

Asociación Española de Normalización y Certificación. UNE. 2013. Estructuras de madera. Madera laminada encolada y madera maciza encolada. Requisitos. UNE-EN 14080. 2013. Asociación Española de Normalización y Certificación: Madrid, España.

Asociación Española de Normalización y Certificación. UNE. 2012. Estructuras de madera. Madera aserrada y madera laminada encolada para uso estructural. Determinación de algunas propiedades físicas y mecánicas. UNE-EN 408. 2012. Asociación Española de Normalización y Certificación: Madrid, España.

Asociación Española de Normalización y Certificación. UNE. 2004. Contenido de humedad de una pieza de madera aserrada. Parte 1: Determinación por el método de secado en estufa. UNE-EN 13183-1. 2004. Asociación Española de Normalización y Certificación: Madrid, España.

Asociacao Brasileira da Normas Técnicas. 1997. Projeto de estruturas de madeira. NBR 7190. 1997. ABNT: Rio de Janeiro, Brasil. 
Aicher, S.; Ahmad, Z.; Hirsch, M. 2018. Bondline shear strength and wood failure of European and tropical hardwood glulams. Eur J Wood Prod 76(4): 1205-1222.

Ayarkwa, J.; Y. Hirashima, Y.; Sasaki, Y. 2000. Effect of finger geometry and end pressure on the flexural properties of finger-jointed tropical African hardwoods. Forest Prod J 50 (11/12): 53-63.

Baño, V.; Moya, L.; O’Neill, H.; Cardoso, A.; Cagno, M.; Cetrangolo, G.; Domenech, L. 2016. Documentos técnicos base para la normalización de estructuras y construcción con madera. Fondo Industrial PR No: 3823/013, Montevideo, Uruguay. 156 p. Disponible en: $<\underline{\text { https://www.fing.edu.uy/sites/default/ }}$ files/2016/25086/1.\%20Baño\%20et\%20al.2015-PrFI-3823-013 InformeFinal.pdf.> (01/05/2019).

Bourscheid, C.; Terezo, R.; Stüp, A.; Vanzella, D. 2015. Desempenho mecânico de madeira laminada colada de Eucalyptus spp. II Congresso Brasileiro de Ciência e Tecnologia da Madeira, Belo Horizonte, Brasil, 10p.

Bustos, C.; Beauregard, R.; Mohammad, M.; Hernandez, R. 2003. Structural performance of finger-jointed black spruce lumber with different joint configurations. Forest Prod J 53 (9): 72-76.

Calil Neto, C.; Christoforo, A.L.; Ribeiro Filho, S.L.M.; Rocco Lahr, F.A.; Calil Junior, C. 2014. Avaliacao da resistencia ao cisalhamento e a delaminacao em madeira laminada colada. Ciencia Florestal 24(4):989-996.

Castro, G.; Paganini, F. 1997. Parameters affecting end finger joint performance in poplar wood. Proceedings of the International Conference of IUFRO S 5.02. Timber Engineering. Copenhagen, Denmark, $10 \mathrm{p}$.

Contreras, W.; Valero, S.W.; Thomson, E.; Owen, M.E. 2007. Determinación de los esfuerzos de vigas laminadas de pino caribe (Pinus caribaea var. hondurensis) encoladas con adhesive de isocianato (MDI). Maderas-Cienc Tecnol 9(3): 285-298.

De Almeida, H.; Cavalheiro, R.S.; Macedo, L.B.; Calil Neto, C.; Christoforo, A.L.; Calil Junior, C.; Rocco Lahr, F.A. 2014. Evaluation of quality in the adhesion of glued laminated timber (Glulam) of Paricá and Lyptus wood species. International Journal of Materials Engineering 4(3): 114-118.

Domenech, L.; Baño, V.; Cetrangolo, G.; Morquio, A. 2016. Rehabilitación de una cubierta de madera: proyecto y reparación. Congreso Euro-Americano REHABEND, Burgos, España, 8p.

Franke, S.; Marto, J. 2014. Investigation of Eucalyptus globulus wood for the use as an engineered material. World Conference on Timber Engineering, Quebec, Canadá, 8p.

Instituto Argentino de Normalización y Certificación. IRAM. 2015. Madera laminada encolada estructural. Parte 1: Clases de resistencia y requisitos de fabricación y de control. IRAM 9660-1. 2015. Buenos Aires, Argentina.

Instituto Argentino de Normalización y Certificación. IRAM. 2015. Madera laminada encolada estructural. Clasificación de tablas por resistencia, Parte 2: Tablas de Eucalyptus grandis. IRAM 9662-2. 2015. Buenos Aires, Argentina.

Lara-Bocanegra, A.J.; Majano-Majano, A.; Crespo, J.; Guaita, M. 2017. Finger-jointed Eucalyptus globulus with 1C-PUR adhesive for high performance engineered laminated products. Construction and Building Materials 135: 528-537.

Özcifci, A.; Yapici, F. 2008. Structural performance of the finger-jointed strength of some wood species with different joint configurations. Construction and Building Materials 22(7):1543-1550.

Piter, J.C.; Cotrina, A.D.; Sosa Zitto M.A.; Stefani, P.M.; Torrán, E.A. 2007. Determination of characteristic strength and stiffness values in glued laminated beams of Argentinean Eucalyptus grandis according to European standards. Holz Roh- Werkst 65: 261-266.

Vega, A.; Baño, V.; Pérez Gomar, C.; Cardoso, A.; Godoy, D.; O’Neill, H.; Moya, L. 2017. Evaluación de la resistencia de uniones dentadas finger-joint con madera de Eucalyptus grandis de procedencia 
uruguaya. II Congreso Latinoamericano de Estructuras de Madera y II Congreso Iberoamericano de la Madera en la Construcción. Junín, Argentina, 7p. 